INPLASY

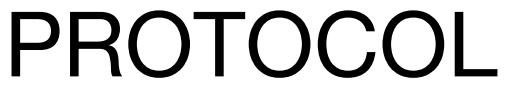

To cite: Lamberg et al. Ocular side effects of systemic isotretinoin: a systematic review and meta-analysis. Inplasy protocol 202160042. doi:

10.37766/inplasy2021.6.0042

Received: 14 June 2021

Published: 14 June 2021

Corresponding author:

Olivia Lamberg

lamberol@med.umich.edu

Author Affiliation:

University of Michigan Medical

School; Michigan Medicine

Department of Dermatology

Support: University of Michigan.

Review Stage at time of this submission: Formal screening of search results against eligibility criteria.

Conflicts of interest:

None declared.

\section{Ocular side effects of systemic} isotretinoin: a systematic review and meta-analysis

Lamberg, O1; Strome, A2; Jones, F3; Mleczek, J4; Vanderboll, K5; Helfrich, $\mathrm{Y}^{6}$.

Review question / Objective: What is the association between ocular side effects and patients receiving systemic isotretinoin?

Condition being studied: Ocular adverse events/side effects.

Eligibility criteria: Inclusion criteria: 1. RCT, case-control, cohort, or case reports* in which the patients received systemic isotretinoin as the medication used. 2. Report the occurrence of ocular or visual side effects 3. Year published: 1970 - present 4. Written in English; Exclusion criteria: 1. Duplicate publications 2. Conference papers, meta-analysis, systematic reviews 3 . Studies that do not mention ocular side effects, either their occurrence or the lack thereof 4 . Written in a language other than English 5. Did not involve human subjects ${ }^{*}$ Case reports will be included for separate analysis for narrative section of extremely rare ocular side effects. Pertinently, it will not be included in the meta-analysis section. This section will just be comprised of RCTs, case-control and/ or cohort studies.

INPLASY registration number: This protocol was registered with the International Platform of Registered Systematic Review and Meta-Analysis Protocols (INPLASY) on 14 June 2021 and was last updated on 14 June 2021 (registration number INPLASY202160042).

\section{INTRODUCTION}

Review question / Objective: What is the association between ocular side effects and patients receiving systemic isotretinoin?
Condition being studied: Ocular adverse events/side effects.

\section{METHODS}

Search strategy: Searched in 3 databases: PubMed, Embase, Scopus PubMed ("Isotretinoin"[Mesh] OR "13-cis-Retinoic 
Acid"[tiab] OR Accutane[tiab] OR Isotretinoin[tiab] OR Roaccutane[tiab] OR "Ro 4-3780"[tiab]) AND ("Eye"[MeSH] OR "Eye Diseases"[Mesh] OR "Ophthalmology" [Mesh] OR "Optometry"[Mesh] OR "Vision, Ocular"[MeSH] OR eye[tiab] OR eyes[tiab] OR ocular[tiab] OR ophthalmic[tiab] OR ophthalmologis t [ $t i a b$ ] OR ophthalmologists [ tiab] OR ophthalmology[tiab] OR optometry[tiab] OR optometric[tiab] OR optometrist[tiab] OR optometrists[tiab] OR retina[tiab] OR retinal[tiab] OR retinas[tiab] OR vision[tiab] OR optic[tiab] OR optics[tiab] OR cornea[tiab] OR conjunctiva[tiab] OR blindness[tiab] OR refractive[tiab] OR refraction[tiab] OR keratitis[tiab] OR keratoconus[tiab] OR vascularization[tiab] OR blepharoconjunctivitis[tiab] OR "subconjunctival hemorrhage"[tiab] OR "subconjunctival hemorrhages"[tiab] OR ptosis[tiab] OR sicca[tiab] OR "idiopathic intracranial hypertension"[tiab] OR "visual field defects"[tiab] OR "dark adaptation"[tiab] OR photophobia[tiab] OR diplopia[tiab] OR iritis[tiab] OR mydriasis[tiab] OR angioedema[tiab] OR glaucoma[tiab] OR scleritis[tiab] OR epiphora[tiab]) Embase ('isotretinoin'/exp OR (isotretinoin OR "13-cis-Retinoic Acid" OR Accutane OR Roaccutane OR "Ro 4-3780"):ti,ab) AND ((eye OR eyes OR ocular OR ophthalmic OR ophthalmologist OR ophthalmologists OR ophthalmology OR optometry OR optometric OR optometrist OR optometrists OR retina OR retinal OR retinas OR vision OR optic OR optics OR cornea OR conjunctiva OR blindness $O R$ refractive $O R$ refraction $O R$ keratitis OR keratoconus OR vascularization $O R$ blepharoconjunctivitis OR "subconjunctival hemorrhage" OR "subconjunctival hemorrhages" OR ptosis OR sicca OR "idiopathic intracranial hypertension" OR "visual field defects" OR "dark adaptation" OR photophobia OR diplopia OR iritis OR mydriasis OR angioedema OR glaucoma OR scleritis OR epiphora):ti,ab) Scopus TITLEABS((isotretinoin OR "13-cis-Retinoic Acid" OR Accutane OR Roaccutane OR "Ro 4-3780") AND (eye OR eyes OR ocular OR ophthalmic OR ophthalmologist OR ophthalmologists OR ophthalmology OR optometry OR optometric OR optometrist OR optometrists OR retina OR retinal OR retinas OR vision OR optic OR optics OR cornea OR conjunctiva OR blindness OR refractive OR refraction $O R$ keratitis $O R$ keratoconus $O R$ vascularization $O R$ blepharoconjunctivitis OR "subconjunctival hemorrhage" OR "subconjunctival hemorrhages" OR ptosis OR sicca OR "idiopathic intracranial hypertension" OR "visual field defects" OR "dark adaptation" OR photophobia OR diplopia OR iritis OR mydriasis OR angioedema OR glaucoma OR scleritis OR epiphora)).

Participant or population: Patients $\mathbf{0 - 1 0 0}$ years old, any gender, any sexual orientation, with no geographical constraints for any indication who have used isotretinoin.

Intervention: Systemic isotretinoin.

Comparator: For the majority of our studies the comparator group is the population studied prior to the isotretinoin intervention.

Study designs to be included: RCT, casecontrol, cohort, case report.

Eligibility criteria: Inclusion criteria: 1. RCT, case-control, cohort, or case reports* in which the patients received systemic isotretinoin as the medication used. 2. Report the occurrence of ocular or visual side effects 3. Year published: 1970 present 4. Written in English; Exclusion criteria: 1. Duplicate publications 2. Conference papers, meta-analysis, systematic reviews 3 . Studies that do not mention ocular side effects, either their occurrence or the lack thereof 4. Written in a language other than English 5. Did not involve human subjects *Case reports will be included for separate analysis for narrative section of extremely rare ocular side effects. Pertinently, it will not be included in the meta-analysis section. This section will just be comprised of RCTs, case-control and/or cohort studies. 
Information sources: Electronic databases (3) - PubMed, Scopus, Embase.

Main outcome(s): Meta-analysis: Study design type - options: randomized controlled trial, case-control study, cluster randomized controlled trial, prospective cohort study, retrospective cohort study, other. Number of patients studied. Age: (mean, $\mathrm{Cl}, \mathrm{N}$ ) or (mean, SD, N) and range. specify which data type; Race (mean, $\mathrm{Cl}, \mathrm{N}$ ) or (mean, SD, N) specify which data type; Sex (mean, Cl, N) or (mean, SD, N) specify which data type; Dx being treated with Isotretinoin; Dosage of Isotretinoin $(\mathrm{mg} / \mathrm{kg}$ / day); Treatment duration: Length of time on Isotretinoin (weeks); Concomitant therapy (other medications, light therapy, etc.); Ocular side effect(s); Length of time into isotretinoin treatment that side effect occurred (weeks); Length of time that the side effects lasted (weeks); Length of follow-up time (months) (how long did the case report follow this patient, may not be reported; Discontinuation of Isotretinoin due to side effects? $\mathrm{Y} / \mathrm{N}$. If yes, at how many weeks into isotretinoin treatment; Treatments to address ocular side effects. $\mathrm{Y} / \mathrm{N}$. If yes, describe. Efficacy/outcome of treatments to address ocular side effects; risk of bias assessment. Case Report: Dx being treated with Isotretinoin; Dosage of Isotretinoin ( $\mathrm{mg} / \mathrm{kg} / \mathrm{day})$; Treatment duration: Length of time on Isotretinoin (weeks); Concomitant therapy (other medications, light therapy, etc.); Ocular side effect(s); Length of time into isotretinoin treatment that side effect occurred (weeks); Length of time that the side effects lasted (weeks); Length of follow-up time (months) (how long did the case report follow this patient, may not be reported); Discontinuation of Isotretinoin due to side effects? $\mathrm{Y} / \mathrm{N}$. If yes, at how many weeks into isotretinoin treatment; Treatments to address ocular side effects. $\mathrm{Y} / \mathrm{N}$. If yes, describe. Efficacy/outcome of treatments to address ocular side effects.

Data management: For the abstract and full-text screening phases we will use Covidence. We will use the risk of bias tool for meta-analysis papers. We will use excel for data analysis.
Quality assessment / Risk of bias analysis: For included RCTs, study quality was assessed using the Cochrane Collaboration's Risk of Bias Assessment Tool according to specific study features. The study features will include random sequence generation, allocation concealment, blinding of participants and personnel, blinding of outcome assessment, incomplete outcome data, and selective outcome reporting. Each study feature will be assigned either high, low or unclear risk of bias. An overall assessment of bias will be performed based on the criteria that had the strongest impact on efficacy and adverse events data. This will also be reported as high, low or unclear risk of bias.

Strategy of data synthesis: The main outcome of interest is the likelihood of patients acquiring specific ocular side effects while taking systemic isotretinoin. Since not all of the studies included will have a control group of patients not taking isotretinoin, our study population is solely patients taking systemic isotretinoin. We will be examining the incidence rates of various ocular side effects. Regarding occurrence of specific ocular side effects: For each study, the percent of patients who experienced each specific ocular side effect will be calculated. We will pool these percentages across studies. We will also compile percentages for each specific ocular side effect based on subgroups listed in the next section. These will then be compared to each other for subgroup analysis. These percentages will also be compared to the total population pooled percentages to assess if these subgroups result in any significant differences from the total population. To quantify time into taking isotretinoin that a specific ocular side effect occurred: If noted in the study, we will compile the average time and $95 \%$ $\mathrm{Cl}$ into taking systemic isotretinoin that a specific ocular side effect occurred. These will be pooled across studies amongst each eye side effect.

Subgroup analysis: We are interested in how dosage of isotretinoin may have played a role in the occurrence of ocular 
toxicities. The total population will be divided into subsets of isotretinoin dosages with adequate power in each group. The above methodology will then be conducted.

Sensitivity analysis: We will not be performing a sensitivity analysis given that our data is limited to incidence rates.

Language: English.

Country(ies) involved: United States.

Keywords: isotretinoin; accutane; 13-cisretinoic acid; ocular side effects.

Contributions of each author:

Author 1 - Olivia Lamberg - Created the project, managed the data, served as the team leader, and drafted the manuscript.

Email: lamberol@med.umich.edu

Author 2 - Arianna Strome - Helped with project management, extracted data, supported in drafting the manuscript.

Email: stromeal@med.umich.edu

Author 3 - Foster Jones - Participated in paper selection and data extraction and well as specifically worked on case-report data synthesis.

Email: fozzy@umich.edu

Author 4 - Julia Mleczek - Participated in paper selection and data extraction and well as specifically worked on case-report data synthesis.

Email: jmleczek@umich.edu

Author 5 - Kathryn Vanderboll - Devised and participated in the methodology of the project.

Email: kvanderb@umich.edu

Author 6 - Yolanda Helfrich - Served as the PI on the project. Supported with creating the project concept, project management, and advising on the manuscript draft.

Email: yolanda@med.umich.edu 